\title{
INTÉRÊT DU RECUEIL CHIRURGICAL DE SPERMATOZOÏDES DÉFÉRENTIELS DANS CERTAINES INDICATIONS MASCULINES DE PMA (IAC,FIV) *
}

\author{
Didier Delafontaine
}

74, avenue Paul Doumer - 75116 PARIS

\begin{abstract}
INTEREST OF OPERATIVE RECOVERY OF DEFERENTIAL SPERM IN SOME MALE INDICATIONS FOR ASSISTED PROCREATION (AID, IVF). Two pregnancies have been obtained after assisted procreation using deferential sperm : one by IVF, one by AIC. The indications of this technique are : obstruction of deferens or of ejaculatory ductus, some anejaculations or retrograde ejaculations resistant to classical treatments. Key-words : deferens, anejaculation, retrograde ejaculation, aspermia, AIC, IVF.

ANDROLOGIE, 1991, 1 : 22-23.
\end{abstract}

L'obtention de spermatozoïdes mobiles en nombre suffisant à partir d'un éjaculat dépend de la fabrication (testicules), de la maturation (épididymes), du stockage (anses épididymodéférentielles) et de l'évacuation de ces spermatozoïdes (canaux déférents, canaux éjaculateurs et sphincter lisse vésical).

Une solution nouvelle, la ponction déférentielle, est proposée pour les absences d'évacuation avec conservation du stockage dans le cadre des sténoses des déférents ou des canaux éjaculateurs, des anéjaculations et des éjaculations rétrogrades rebelles aux traitements classiques. $(7,8)$

\section{MATÉRIEL ET MÉTHODES}

1 - Patients (tableau ${ }^{\circ} 1$ ) : Huit hommes (4 anéjaculations et 4 éjaculations rétrogrades) sont entrés dans un programme de ponction déférentielle. Chez les 4 éjaculateurs rétrogrades, toute tentative de récupération des spermatozoïdes après miction avec contrôle de l'osmoralité et du $\mathrm{pH}$ ont échoué.

2 - Techniques opératoire et biologique : Le prélèvement déférentiel s'effectue sous anesthésie locale selon une technique macrochirurgicale après répérage du déférent le plus haut possible dans le scrotum ; incision oblique d'un centimètre en regard du canal déférent,

* Présenté au $8^{\text {eme }}$ congrès de la SALF Sousse, 6-8 Septembre 1990. dissection du déférent, vasotomie transversale jusqu'à la lumière ; cathétérisme de la lumière vers l'anse épididymo-déférentielle ; lavage de l'anse épididymo-déférentielle par injectionréaspiration de B2 de Menezo; examen des recueils par le biologiste qui sont ensuite techni- qués par centrifugation, migration ou sur gradient de Percoll en vue d'une IAC ou d'une FIV, et éventuellement congelés pour une ou plusieurs PMA ultérieures, vaso-vasostomie à l'aide d'un tuteur de catgut 000 selon la technique de Redman (10).

\begin{tabular}{|c|c|c|c|c|c|c|c|c|c|}
\hline \multicolumn{10}{|c|}{ TABLEAU 1 : PMA AVEC SPERME DÉFÉRENTIEL : EXPÉRIENCE PERSONNELLE } \\
\hline $\begin{array}{l}\text { Auteurs } \\
\text { (ref) }\end{array}$ & $\mathrm{N}$ cas & Etiologie & $\begin{array}{l}\text { Technique } \\
\text { chinurgicale }\end{array}$ & $\begin{array}{l}\text { Fluide de } \\
\mathrm{N}(\mathrm{M})\end{array}$ & $\begin{array}{l}\text { ponction } \\
\operatorname{Mob}(\%)\end{array}$ & PMA & Cycles & Grossesse & Remarques \\
\hline $\begin{array}{c}\text { DELAFONTANE } \\
1988-1990 \\
(7)\end{array}$ & 4 & $\begin{array}{c}A \\
A \\
A \\
A \\
\text { (paraplégie) }\end{array}$ & $\begin{array}{l}\text { macro } \\
\text { macro } \\
\text { macro } \\
\text { macro }\end{array}$ & $\begin{array}{c}20 \\
16-18 \\
8-12 \\
\text { quelques }\end{array}$ & $\begin{array}{c}80 \\
80-90 \\
45-50 \\
-\end{array}$ & $\begin{array}{l}\text { FVV } \\
\text { FIV } \\
\text { IAC } \\
-\end{array}$ & $\begin{array}{l}1 \\
2 \\
2 \\
1\end{array}$ & $\begin{array}{c}\text { gémellaire } \\
0 \\
0 \\
0\end{array}$ & $\begin{array}{l}\text { acct } 2 \text { garçons } \\
\text { adoption } \\
- \\
\text { grossesse après } 2 \\
\text { cycle d'IAD }\end{array}$ \\
\hline $\begin{array}{c}\text { DELAFONTAINE } \\
1990 \\
(8)\end{array}$ & 4 & $\begin{array}{l}\text { ER } \\
\text { ER } \\
\text { ER } \\
\text { ER }\end{array}$ & $\begin{array}{l}\text { macro } \\
\text { macro } \\
\text { macro } \\
\text { macro }\end{array}$ & $\begin{array}{c}51 \\
6,5 \\
40 \\
7 \\
8-20\end{array}$ & $\begin{array}{c}50 \\
70 \\
80 \\
90 \\
40.60\end{array}$ & $\begin{array}{l}\text { IAC } \\
\text { IAC } \\
\text { IAC } \\
\text { FIV } \\
\text { IAC }\end{array}$ & $\begin{array}{l}1 \\
4 \\
3 \\
1 \\
4\end{array}$ & $\begin{array}{c}\text { gémellaire } \\
0 \\
0 \\
0 \\
0\end{array}$ & $\begin{array}{l}\text { acct } 2 \text { garcons } \\
\text { congélation } 6 \mathrm{p} \\
\text { congélation } 5 p \\
(2 \mathrm{ov} \rightarrow 1 \mathrm{emb})\end{array}$ \\
\hline
\end{tabular}

A: Anéjaculation - ER: Ejaculation rétrograde - SD:Sténose déférentielle

\begin{tabular}{|c|c|c|c|c|c|c|c|c|c|}
\hline \multicolumn{10}{|c|}{ TABLEAU 2 : PMA AVEC SPERME DÉFÉRENTIEL : REVUE DE LA LITTÉRATURE } \\
\hline $\begin{array}{l}\text { Auteurs } \\
\text { (ref) }\end{array}$ & $\mathrm{N}$ cas & Etiologie & $\begin{array}{l}\text { Technique } \\
\text { chirurgicale }\end{array}$ & $\begin{array}{l}\text { Fluide } \\
N(M)\end{array}$ & $\begin{array}{l}\text { ponction } \\
M o b(\%)\end{array}$ & PMA & Cycles & Grossesse & Remarques \\
\hline $\begin{array}{l}\text { COLPI et al. } \\
\text { 1983.1986 }\end{array}$ & $\begin{array}{c}1 \\
1 \\
11\end{array}$ & $\begin{array}{l}S D \\
\text { SD } \\
\text { SD }\end{array}$ & $\begin{array}{l}\text { réservoir } \\
\text { réservoir } \\
\text { réservoirs }\end{array}$ & $\begin{array}{c}145 \\
100 \\
? \\
?\end{array}$ & $\begin{array}{c}10-39 \\
10 \\
? \\
?\end{array}$ & $\begin{array}{c}\text { IAC } \\
\text { FIV } \\
\text { IAC } \\
\text { Echec }\end{array}$ & $\begin{array}{c}? \\
1 \\
15 \\
-\end{array}$ & $\begin{array}{l}0 \\
0 \\
0 \\
\text {. }\end{array}$ & $\begin{aligned} 20 \mathrm{v} . & \rightarrow 2 \mathrm{emb} . \\
& - \\
& - \\
& -\end{aligned}$ \\
\hline $\begin{array}{l}\text { PRYOR et al. } \\
1984\end{array}$ & 1 & SD & $\begin{array}{l}\text { macro } \\
\text { réservoir }\end{array}$ & $\begin{array}{l}17,7 \\
20\end{array}$ & $\begin{array}{l}70 \\
?\end{array}$ & $\mathrm{FIV}$ & 1 & gémellaire & $\begin{array}{l}\mathrm{FCS}(11 \mathrm{SA}) \\
\text { Port-a-cath }\end{array}$ \\
\hline $\begin{array}{c}\text { BERGER et al. } \\
1986\end{array}$ & $\begin{array}{l}2 \\
1\end{array}$ & $\begin{array}{l}\text { A } \\
\text { ER }\end{array}$ & $\begin{array}{l}\text { micro } \\
\text { micro }\end{array}$ & $\begin{array}{l}10-60 \\
35-60\end{array}$ & $\begin{array}{c}50-65 \\
50\end{array}$ & $\begin{array}{l}\text { IAC } \\
\text { IAC }\end{array}$ & $\begin{array}{c}1-3 \\
1\end{array}$ & $\begin{array}{l}0 \\
0\end{array}$ & \\
\hline $\begin{array}{c}\text { BRINDLEY et al. } \\
1986\end{array}$ & 7 & SD & réservoirs & $\begin{array}{c}20-140 \\
120 \\
5 \\
5 \\
30-100 \\
-\end{array}$ & $\begin{array}{c}1-10 \\
30 \\
- \\
30 \\
1-30 \\
-\end{array}$ & $\begin{array}{c}\text { 3IAC } \\
\text { 1FIV } \\
\text { 1 Echec } \\
\text { IAC } \\
\text { I FIV } \\
\text { 5Echecs }\end{array}$ & $\begin{array}{l}? \\
1 \\
\dot{?} \\
1 \\
\text { ? }\end{array}$ & $\begin{array}{l}1 \\
0 \\
. \\
1 \\
-\end{array}$ & acct fille nle \\
\hline BUSTILLOet al. & 1 & A & macro & $?$ & 50 & IAC & 2 & 1 & $\begin{array}{l}\text { acct césarienne } \\
\text { garçon nl }\end{array}$ \\
\hline
\end{tabular}




\section{RESULTATS}

Deux grossesses ont été obtenues dans notre série personnelle :

- pour le premier couple, l'indication était une anéjaculation primaire rebelle à la sexothérapie et au vibromasseur, le couple consultant au départ pour IAD. La ponction déférentielle a permis d'obtenir 20 millions de spermatozoïdes (80\% mobiles); une tentative de FIV a permis d'obtenir cinq embryons dont trois ont été replacés et deux congelés. Une grossesse gémellaire a évolué normalement : naissance de 2 garçons bien portants. (7)

- pour le deuxième couple, l'indication était une éjaculation rétrograde (orchitectomie pour néoplasme du testicule et lymphadénectomie) rebelle au traitement classique (recueil de spermatozoïdes dans les urines après contrôle du $\mathrm{PH}$ et de l'osmolarité), la demande initiale était une FIV-D après échec de cinq cycles d'IAD à l'étranger. La ponction déférentielle a recueilli 5 millions de spermatozoïdes dont $50 \%$ de mobiles: une IAC a été réalisée et onze paillettes à $0,25 \mathrm{ml}$ ont été congelées. La grossesse a suivi le premier cycle d'IAC. Accouchement de deux garçons bien portants. (8)

\section{DISCUSSION}

Bowen (2) a montré, chez le chat, que des spermatozoïdes non capacités in vivo obtenus après ponction déférentielle étaient capables de féconder in vitro des ovocytes (taux de clivage 79\%)(2). Chez l'homme, les premières tentatives de recueil de spermatozoïdes déférentiels ont été réalisées dans le cadre de sténoses déférentielles: COLPI en 1983 obtient par une technique chirurgicale utilisant un réservoir 145 millions de spermatozoïdes dont la mobilité est de 10 à $39 \%$. $(5,6)$
PRYOR publie la première grossesse, l'indication est une sténose déférentielle bilatérale. Après un recueil déférentiel de 18 millions de spermatozoïdes (mobilité $70 \%$ ), une tentative de FIV aboutit à une grossesse gémellaire qui n'évoluera pas au delà de 11 semaines d'aménorrhée. (9) BERGER, BRINDLEY et BUSTILLO étendent les indications du prélèvement déférentiel aux anéjaculations et éjaculations rétrogrades rebelles au traitement classique. $(1,3,4)$. Deux grossesses sont obtenues dans la série de Brindley ; la première dans le cadre d'une anéjaculation, la seconde dans le cadre d'une sténose déférentielle bilatérale. (3) Bustillo publie une grossesse (un enfant né normal) dans le cadre d'une anéjaculation. (4) Les données de la littérature sont regroupées dans le tableau $\mathrm{n}^{\circ} 2$.

Nous rapportons en 1990 la première grossesse évolutive dans le cadre d'une éjaculation rétrograde rebelle aux traitements classiques. (8)

\section{CONCLUSION}

Malgré des résultats encore modestes, ces nouveaux modes de prélèvement des spermatozoïdes manifestement peu connus ont l'intérêt de pouvoir éviter l'IAD ou l'adoption pour ces couples étiquetés jusqu'alors à tort, définitivement stériles.

\section{REFERENCES}

I Berger R.E., Muller C.H., Smith D., Forster M., Moore D., Mc Intosh R., Stewart B. Operative recovery of vasal sperm from anejaculatory men : preliminary report. The Journal of Urology, 1986, 135 : 948-950.

2 Bowen R.A. Fertilization in vitro of feline ova by spermatozoa from the ductus deferens. Biology of Reproduction 1977, 17: 144-147.

3 Brindley G.S., Scott G.I., Hendry W.F. Vas cannulation with implanted sperm reservoirs for obstructive azoospermia or ejaculatory failure. British Jounal of Urology, 1986, $58: 721-723$.
4 Bustillo M. Rajfer J. Pregnancy following insemination with sperm aspirated directly from vas deferens. Fertility Sterility, 1986, 46 : 144-146.

5 Colpi G.M., Zanollo A., Lange A., Farina U., Beretta G. Artificial spermatocele inserted on the vas deferens: a clinical report. Acta Europaea Fertilatis, 1983, 14 : 203-208.

6 Colpi G.M., Zanollo A., Van Kooij R.J., Campana A., Balerna M. Fertilization of human oocytes by spermatozoa from an artificial spermatocele. Acta europaea fertilatis, 1986, $17: 221-223$.

7 Delafontaine D. Intérêt de la ponction déférentielle dans certaines indications masculines de FIV. Communication aux $16^{c}$ journées de fertilité orthogénie. Paris, 11 novembre 1988.

8 Delafontaine D., Simeon S., Mugniot-Bellamy S., Menard M.N. Intérêt de la ponction déférentielle dans certains indications masculines de PMA (IAC, FIV). $5^{\circ}$ journées de périconceptologie, GEFF, CECOS, Bordeaux, mai 1990.

9 Pryor J., Parsons J., Goswamy R., Matson P., Vaid P., Wilson L., Whitehead M. In vitro fertilisation for men with obstructive azoospermia. Lancet, 1984, sept. 29 , 762.

10 Redman J.F. Clinical experience with vasovasostomy utilizing absorbable intravasalstent. Urology 1982, 20, 59-61.

Résumé : Deux grossesses ont été obtenues par procréation médicalement assistée utilisant des spermatozoïdes déférentiels : une par fécondation in vitro et l'autre par IAC. Les indications de cette technique sont : les oblitérations des déférents ou des canaux éjaculateurs, certaines anéjaculations ou éjaculations rétrogrades rebelles aux traitements classiques. Mots clés : Déférent, anéjaculation, éjaculation rétrograde, aspermie, IAC, FIV.

Andrologie, 1991, 1 : 22-23 